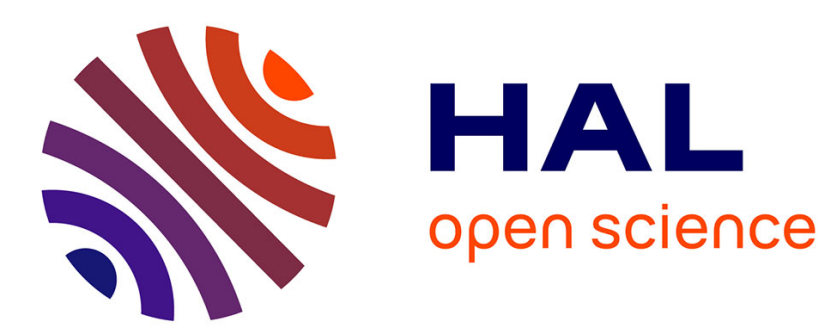

\title{
Asymptotic Behavior of the Scharfetter-Gummel Scheme for the Drift-Diffusion Model
}

Marianne Chatard

\section{To cite this version:}

Marianne Chatard. Asymptotic Behavior of the Scharfetter-Gummel Scheme for the Drift-Diffusion Model. Finite Volumes for Complex Applications VI, Jun 2011, Prague, Czech Republic. pp.235 243, 10.1007/978-3-642-20671-9_25. hal-01472511

\section{HAL Id: hal-01472511 https://hal.science/hal-01472511}

Submitted on 20 Feb 2017

HAL is a multi-disciplinary open access archive for the deposit and dissemination of scientific research documents, whether they are published or not. The documents may come from teaching and research institutions in France or abroad, or from public or private research centers.
L'archive ouverte pluridisciplinaire HAL, est destinée au dépôt et à la diffusion de documents scientifiques de niveau recherche, publiés ou non, émanant des établissements d'enseignement et de recherche français ou étrangers, des laboratoires publics ou privés. 


\title{
Asymptotic Behavior of the Scharfetter-Gummel Scheme for the Drift-Diffusion Model
}

Marianne CHATARD

\begin{abstract}
The aim of this work is to study the large-time behavior of the ScharfetterGummel scheme for the drift-diffusion model for semiconductors. We prove the convergence of the numerical solutions to an approximation of the thermal equilibrium. We also present numerical experiments which underline the preservation of long-time behavior.
\end{abstract}

Key words: Drift-diffusion system, finite volume scheme, thermal equilibrium. MSC2010: 65M08, 76X05, 82D37.

\section{Introduction}

In the modeling of semiconductor devices, the drift-diffusion system is widely used as it simplifies computations while giving an accurate description of the device physics.

Let $\Omega \subset \mathbb{R}^{d}(d \geq 1)$ be an open and bounded domain describing the geometry of the semiconductor device. The isothermal drift-diffusion system consists of two continuity equations for the electron density $N(x, t)$ and the hole density $P(x, t)$, and a Poisson equation for the electrostatic potential $V(x, t)$ :

$$
\begin{cases}\partial_{t} N-\operatorname{div}(\nabla N-N \nabla V)=0 & \text { on } \Omega \times(0, T), \\ \partial_{t} P-\operatorname{div}(\nabla P+P \nabla V)=0 & \text { on } \Omega \times(0, T), \\ \lambda^{2} \Delta V=N-P-C & \text { on } \Omega \times(0, T),\end{cases}
$$

where $C(x)$ is the doping profile, which is assumed to be a given datum, and $\lambda$ is the Debye length arising from the scaling of the physical model. We supplement these

Marianne CHATARD

Université Blaise Pascal - Laboratoire de Mathématiques UMR 6620 - CNRS - Campus des Cézeaux, B.P. 8002663177 Aubière cedex, e-mail: Marianne.Chatard@math.univ-bpclermont.fr 
equations with initial conditions $N_{0}(x)$ and $P_{0}(x)$ and physically motivated boundary conditions: Dirichlet boundary conditions $\bar{N}, \bar{P}$ and $\bar{V}$ on ohmic contacts $\Gamma^{D}$ and homogeneous Neumann boundary conditions on insulating boundary segments $\Gamma^{N}$. There is an extensive literature on numerical schemes for the drift-diffusion equations: finite difference methods, finite elements methods, mixed exponential fitting finite elements methods, finite volume methods (see [1]). The Scharfetter-Gummel scheme is widely used to approximate the drift-diffusion equations in the linear case. It has been proposed and studied in [7] and [10]. It preserves steady-state, and is second order accurate in space (see [9]).

The purpose of this paper is to study the large time behavior of the numerical solution given by the Scharfetter-Gummel scheme for the transient linear drift-diffusion model (1). Indeed, it has been proved by H. Gajewski and K. Gärtner in [5] that the solution to the transient system (1) converges to the thermal equilibrium state as $t \rightarrow \infty$ if the boundary conditions are in thermal equilibrium. A. Jüngel extends this result to a degenerate model with nonlinear diffusivities in [8].

The thermal equilibrium is a particular steady-state for which electron and hole currents, namely $\nabla N-N \nabla V$ and $\nabla P+P \nabla V$, vanish.

If the Dirichlet boundary conditions satisfy $\bar{N}, \bar{P}>0$ and

$$
\log (\bar{N})-\bar{V}=\alpha_{N} \text { and } \log (\bar{P})+\bar{V}=\alpha_{P} \text { on } \Gamma^{D},
$$

the thermal equilibrium is defined by

$$
\begin{cases}\Delta V^{e q}=\exp \left(\alpha_{N}+V^{e q}\right)-\exp \left(\alpha_{P}-V^{e q}\right)-C & \text { on } \Omega, \\ N^{e q}=\exp \left(\alpha_{N}+V^{e q}\right), P^{e q}=\exp \left(\alpha_{P}-V^{e q}\right) & \text { on } \Omega,\end{cases}
$$

with the same boundary conditions as (1).

Our aim is to prove that the solution of the Scharfetter-Gummel scheme converges to an approximation of the thermal equilibrium as $t \rightarrow+\infty$. Long-time behavior of solutions to discretized drift-diffusion systems have been studied in [5], [2] and [6], using estimates of the energy.

In the sequel, we will suppose that the following hypotheses are fulfilled:

(H1) $\bar{N}, \bar{P}$ are traces on $\Gamma^{D} \times(0, T)$ of functions, also denoted $\bar{N}$ and $\bar{P}$, such that

$\bar{N}, \bar{P} \in H^{1}(\Omega \times(0, T)) \cap L^{\infty}(\Omega \times(0, T))$ and $\bar{N}, \bar{P} \geq 0$ a.e.,

(H2) $N_{0}, P_{0} \in L^{\infty}(\Omega)$ and $N_{0}, P_{0} \geq 0$ a.e.,

(H3) there exist $0<m \leq M$ such that: $m \leq \bar{N}, N_{0}, \bar{P}, P_{0} \leq M$,

(H4) $\bar{N}, \bar{P}$ and $\bar{V}$ satisfy the compatibility condition (2).

\section{Numerical schemes}

In this section, we present the finite volume schemes for the time evolution driftdiffusion system (1) and for the thermal equilibrium (3).

An admissible mesh of $\Omega$ is given by a family $\mathscr{T}$ of control volumes (open and 
convex polygons in 2-D, polyhedra in 3-D), a family $\mathscr{E}$ of edges in 2-D (faces in $3-D)$ and a family of points $\left(x_{K}\right)_{K \in \mathscr{T}}$ which satisfy Definition 5.1 in [4]. It implies that the straight line between two neighboring centers of cells $\left(x_{K}, x_{L}\right)$ is orthogonal to the edge $\sigma=K \mid L$.

In the set of edges $\mathscr{E}$, we distinguish the interior edges $\sigma \in \mathscr{E}_{\text {int }}$ and the boundary edges $\sigma \in \mathscr{E}_{\text {ext }}$. We split $\mathscr{E}_{\text {ext }}$ into $\mathscr{E}_{\text {ext }}=\mathscr{E}_{\text {ext }} \cup \mathscr{E}_{\text {ext }}$ where $\mathscr{E}_{\text {ext }}$ is the set of Dirichlet boundary edges and $\mathscr{E}_{e x t}$ is the set of Neumann boundary edges. For a control volume $K \in \mathscr{T}$, we denote by $\mathscr{E}_{K}$ the set of its edges, $\mathscr{E}_{\text {int }, K}$ the set of its interior edges, $\mathscr{E}_{\text {ext }, K}^{D}$ the set of edges of $K$ included in $\Gamma^{D}$ and $\mathscr{E}_{\text {ext }, K}^{N}$ the set of edges of $K$ included in $\Gamma^{N}$.

The size of the mesh is defined by $\Delta x=\max _{K \in \mathscr{T}}(\operatorname{diam}(K))$.

We denote by $\mathrm{d}$ the distance in $\mathbb{R}^{d}$ and $\mathrm{m}$ the measure in $\mathbb{R}^{d}$ or $\mathbb{R}^{d-1}$.

We also need some assumption on the mesh:

$$
\exists \xi>0 \text { s. t. } \mathrm{d}\left(x_{K}, \sigma\right) \geq \xi \mathrm{d}\left(x_{K}, x_{L}\right) \text { for } K \in \mathscr{T}, \text { for } \sigma=K \mid L \in \mathscr{E}_{i n t, K}
$$

For all $\sigma \in \mathscr{E}$, we define the transmissibility coefficient $\tau_{\sigma}=\frac{\mathrm{m}(\sigma)}{d_{\sigma}}$, where $d_{\sigma}=\mathrm{d}\left(x_{K}, x_{L}\right)$ for $\sigma=K \mid L \in \mathscr{E}_{\text {int }}$ and $d_{\sigma}=\mathrm{d}\left(x_{K}, \sigma\right)$ for $\sigma \in \mathscr{E}_{\text {ext }}$.

Let $\left(\mathscr{T}, \mathscr{E},\left(x_{K}\right)_{K \in \mathscr{T}}\right)$ be an admissible discretization of $\Omega$ and let us define the time step $\Delta t, N_{T}=E(T / \Delta t)$ and the increasing sequence $\left(t^{n}\right)_{0 \leq n \leq N_{T}}$, where $t^{n}=n \Delta t$, in order to get a space-time discretization $\mathscr{D}$ of $\Omega \times(0, T)$. The size of the space-time discretization $\mathscr{D}$ is defined by $\delta=\max (\Delta x, \Delta t)$.

First of all, the initial conditions and the doping profile are approximated by $\left(N_{K}^{0}, P_{K}^{0}, C_{K}\right)_{K \in \mathscr{T}}$ by taking the mean values of $N_{0}, P_{0}$ and $C$ on each cell $K$. The numerical boundary conditions $\left(N_{\sigma}^{n+1}, P_{\sigma}^{n+1}, V_{\sigma}^{n+1}\right)_{n \geq 0, \sigma \in \mathscr{E}_{\text {ext }}^{D}}$ are also given by the mean values of $(\bar{N}, \bar{P}, \bar{V})$ on $\sigma \times\left[t^{n}, t^{n+1}[\right.$.

\subsection{The scheme for the thermal equilibrium}

We compute an approximation $\left(N_{K}^{e q}, P_{K}^{e q}, V_{K}^{e q}\right)_{K \in \mathscr{T}}$ of the thermal equilibrium $\left(N^{e q}\right.$, $\left.P^{e q}, V^{e q}\right)$ defined by (3) with the finite volume scheme proposed by $\mathrm{C}$. ChainaisHillairet and F. Filbet in [2]:

$$
\begin{cases}\lambda^{2} \sum_{\sigma \in \mathscr{E}_{K}} \tau_{\sigma} D V_{K, \sigma}^{e q}=\mathrm{m}(K)\left(\exp \left(\alpha_{N}+V_{K}^{e q}\right)-\exp \left(\alpha_{P}-V_{K}^{e q}\right)-C_{K}\right) & \forall K \in \mathscr{T}, \\ N_{K}^{e q}=\exp \left(\alpha_{N}+V_{K}^{e q}\right), \quad P_{K}^{e q}=\exp \left(\alpha_{P}-V_{K}^{e q}\right) & \forall K \in \mathscr{T}\end{cases}
$$

where for a given function $f$ and $\left(U_{K}\right)_{K \in \mathscr{T}}, D f(U)_{K, \sigma}$ is defined by:

$$
D f(U)_{K, \sigma}= \begin{cases}f\left(U_{L}\right)-f\left(U_{K}\right) & \text { if } \sigma=K \mid L \in \mathscr{E}_{\text {int }, K} \\ f\left(U_{\sigma}\right)-f\left(U_{K}\right) & \text { if } \sigma \in \mathscr{E}_{\text {ext }, K} \\ 0 & \text { if } \sigma \in \mathscr{E}_{\text {ext }, K}\end{cases}
$$


Assuming that the boundary conditions satisfy hypotheses (H1)-(H4), the scheme (4) admits a unique solution (see [2]).

\subsection{The scheme for the transient model}

The Scharfetter-Gummel scheme for the system (1) is defined by:

$$
\begin{cases}\mathrm{m}(K) \frac{N_{K}^{n+1}-N_{K}^{n}}{\Delta t}+\sum_{\sigma \in \mathscr{E}_{K}} \mathscr{F}_{K, \sigma}^{n+1}=0, & \forall K \in \mathscr{T}, \forall n \geq 0, \\ \mathrm{~m}(K) \frac{P_{K}^{n+1}-P_{K}^{n}}{\Delta t}+\sum_{\sigma \in \mathscr{E}_{K}} \mathscr{G}_{K, \sigma}^{n+1}=0, & \forall K \in \mathscr{T}, \forall n \geq 0, \\ \lambda^{2} \sum_{\sigma \in \mathscr{E}_{K}} \tau_{\sigma} D V_{K, \sigma}^{n}=\mathrm{m}(K)\left(N_{K}^{n}-P_{K}^{n}-C_{K}\right), & \forall K \in \mathscr{T}, \forall n \geq 0,\end{cases}
$$

with for all $\sigma \in \mathscr{E}_{K}$

$$
\begin{aligned}
\mathscr{F}_{K, \sigma}^{n+1} & =\tau_{\sigma}\left(B\left(-D V_{K, \sigma}^{n+1}\right) N_{K}^{n+1}-B\left(D V_{K, \sigma}^{n+1}\right) N_{\sigma}^{n+1}\right), \\
\mathscr{G}_{K, \sigma}^{n+1} & =\tau_{\sigma}\left(B\left(D V_{K, \sigma}^{n+1}\right) P_{K}^{n+1}-B\left(-D V_{K, \sigma}^{n+1}\right) P_{\sigma}^{n+1}\right),
\end{aligned}
$$

where $B$ is the Bernoulli function defined by:

$$
B(x)=\frac{x}{e^{x}-1} \text { for } x \neq 0, \quad B(0)=1 .
$$

We consider a fully implicit discretization in time to avoid the restrictive stability condition $\Delta t \leq \lambda^{2} / M$.

Using a fixed point theorem, we can prove the following result:

Theorem 1. Let us assume $(H 1)-(H 4)$ and $C=0$. Then there exists a solution $\left\{\left(N_{K}^{n}, P_{K}^{n}, V_{K}^{n}\right), K \in \mathscr{T}, 0 \leq n \leq N_{T}+1\right\}$ to the scheme (5)-(6)-(7), and moreover we have

$$
0<m \leq N_{K}^{n}, P_{K}^{n} \leq M, \quad \forall K \in \mathscr{T}, \quad \forall n \geq 0
$$

\section{Asymptotic behavior of the Scharfetter-Gummel scheme}

We may now state our main result.

Theorem 2. Let us assume $(H 1)-(H 4)$ and $C=0$. Then solution $\left(N_{\delta}, P_{\delta}, V_{\delta}\right)$ given by the scheme (5)-(6)-(7) satisfies for each $K \in \mathscr{T}$

$$
\left(N_{K}^{n}, P_{K}^{n}, V_{K}^{n}\right) \longrightarrow\left(N_{K}^{e q}, P_{K}^{e q}, V_{K}^{e q}\right) \text { as } n \rightarrow+\infty,
$$


where $\left(N_{K}^{e q}, P_{K}^{e q}, V_{K}^{e q}\right)_{K \in \mathscr{T}}$ is an approximation to the solution of the steady-state equation (3) given by (4).

The proof is based, as in the continuous case (see [5] and [8]), on an energy estimate and a control of its dissipation, given in Proposition 1 which is valid even if $C \neq 0$. Nevertheless to prove rigorously the convergence to equilibrium, we need the uniform lower bound (9) on $N$ and $P$ which holds under the restrictive assumption $C=0$.

In the last section, we perform some numerical experiments and observe a convergence to steady-state even when this condition is not satisfied.

\subsection{Notations and definitions}

For $U=\left(U_{K}\right)_{K \in \mathscr{T}}$, we define the $H^{1}$-seminorm as follows:

$$
|U|_{1, \Omega}^{2}=\sum_{\substack{\sigma \in \mathscr{E}_{i n t} \\ \sigma=K \mid L}} \tau_{\sigma}\left|D U_{K, \sigma}\right|^{2}+\sum_{K \in \mathscr{T}} \sum_{\sigma \in \mathscr{E}_{e x t, K}} \tau_{\sigma}\left|D U_{K, \sigma}\right|^{2}
$$

Since the study of the large time behavior of the scheme (5)-(6)-(7) is based on an energy estimate with the control of its dissipation, let us introduce the discrete version of the deviation of the total energy from the thermal equilibrium:

$$
\begin{aligned}
\mathscr{E}^{n}= & \sum_{K \in \mathscr{T}} \mathrm{m}(K)\left(H\left(N_{K}^{n}\right)-H\left(N_{K}^{e q}\right)-\log \left(N_{K}^{e q}\right)\left(N_{K}^{n}-N_{K}^{e q}\right)\right) \\
& +\sum_{K \in \mathscr{T}} \mathrm{m}(K)\left(H\left(P_{K}^{n}\right)-H\left(P_{K}^{e q}\right)-\log \left(P_{K}^{e q}\right)\left(P_{K}^{n}-P_{K}^{e q}\right)\right) \\
& +\frac{\lambda^{2}}{2}\left|V^{n}-V^{e q}\right|_{1, \Omega}^{2} .
\end{aligned}
$$

Since $s \mapsto H(s)=\int_{1}^{s} \log (\tau) d \tau$ is defined and convex on $\mathbb{R}_{+}$, we have $\mathscr{E} n \geq 0$ for all $n \geq 0$. We also introduce the discrete version of the energy dissipation:

$$
\begin{aligned}
& \mathscr{I}^{n}=\sum_{\substack{\sigma \in \mathscr{E}_{i n t} \\
\sigma=K \mid L}} \tau_{\sigma} \min \left(N_{K}^{n}, N_{L}^{n}\right)\left[D\left(\log \left(N^{n}\right)-V^{n}\right)_{K, \sigma}\right]^{2} \\
& +\sum_{K \in \mathscr{T}} \sum_{\sigma \in \mathscr{E}_{\text {ext }, K}} \tau_{\sigma} \min \left(N_{K}^{n}, N_{\sigma}^{n}\right)\left[D\left(\log \left(N^{n}\right)-V^{n}\right)_{K, \sigma}\right]^{2} \\
& +\sum_{\substack{\sigma \in \mathscr{E}_{i n t} \\
\sigma=K \mid L}} \tau_{\sigma} \min \left(P_{K}^{n}, P_{L}^{n}\right)\left[D\left(\log \left(P^{n}\right)+V^{n}\right)_{K, \sigma}\right]^{2} \\
& +\sum_{K \in \mathscr{T}} \sum_{\sigma \in \mathscr{E}_{e x t}, K} \tau_{\sigma} \min \left(P_{K}^{n}, P_{\sigma}^{n}\right)\left[D\left(\log \left(P^{n}\right)+V^{n}\right)_{K, \sigma}\right]^{2} .
\end{aligned}
$$




\subsection{Energy estimate}

The following Proposition gives the control of energy and dissipation. With this result, Theorem 2 can be proved in the same way as Theorem 2.2 in [2].

Proposition 1. Under hypotheses (H1)-(H4), we have for all $n \geq 0$ :

$$
0 \leq \mathscr{E}^{n+1}+\Delta t \mathscr{I}^{n+1} \leq \mathscr{E}^{n} .
$$

Proof. Firstly, using the convexity of $H$ and (4), we get

$$
\begin{aligned}
\mathscr{E}^{n+1}-\mathscr{E}^{n} \leq & \sum_{K \in \mathscr{T}} \mathrm{m}(K)\left(\log \left(N_{K}^{n+1}\right)-\alpha_{N}-V_{K}^{e q}\right)\left(N_{K}^{n+1}-N_{K}^{n}\right) \\
& +\sum_{K \in \mathscr{T}} \mathrm{m}(K)\left(\log \left(P_{K}^{n+1}\right)-\alpha_{P}+V_{K}^{e q}\right)\left(P_{K}^{n+1}-P_{K}^{n}\right) \\
& +\frac{\lambda^{2}}{2}\left|V^{n+1}-V^{e q}\right|_{1, \Omega}^{2}-\frac{\lambda^{2}}{2}\left|V^{n}-V^{e q}\right|_{1, \Omega}^{2},
\end{aligned}
$$

and then, by adding $V_{K}^{n+1}-V_{K}^{n+1}$ in the two first sums, we have

$$
\mathscr{E}^{n+1}-\mathscr{E}^{n} \leq T_{1}+T_{2}+T_{3},
$$

where

$$
\begin{aligned}
T_{1}= & \sum_{K \in \mathscr{T}} m(K)\left(\log \left(N_{K}^{n+1}\right)-\alpha_{N}-V_{K}^{n+1}\right)\left(N_{K}^{n+1}-N_{K}^{n}\right), \\
T_{2}= & \sum_{K \in \mathscr{T}} m(K)\left(\log \left(P_{K}^{n+1}\right)-\alpha_{P}+V_{K}^{n+1}\right)\left(P_{K}^{n+1}-P_{K}^{n}\right), \\
T_{3}= & \sum_{K \in \mathscr{T}} m(K)\left(V_{K}^{n+1}-V_{K}^{e q}\right)\left(N_{K}^{n+1}-N_{K}^{n}-P_{K}^{n+1}+P_{K}^{n}\right) \\
& +\frac{\lambda^{2}}{2}\left|V^{n+1}-V^{e q}\right|_{1, \Omega}^{2}-\frac{\lambda^{2}}{2}\left|V^{n}-V^{e q}\right|_{1, \Omega}^{2} .
\end{aligned}
$$

Using the scheme (5) and an integration by parts, we get that $T_{3} \leq 0$ and

$$
T_{1}=\Delta t \sum_{\substack{\sigma \in \mathscr{E}_{i n t} \\ \sigma=K \mid L}} \tau_{\sigma} \mathscr{R}_{K, \sigma}^{n+1}+\Delta t \sum_{K \in \mathscr{T}} \sum_{\sigma \in \mathscr{E}_{\text {ext }, K}^{D}} \tau_{\sigma} \mathscr{R}_{K, \sigma}^{n+1}
$$

where for $\sigma=K \mid L$,

$$
\mathscr{R}_{K, \sigma}^{n+1}=\left(D \log \left(N^{n+1}\right)_{K, \sigma}-D V_{K, \sigma}^{n+1}\right)\left(B\left(-D V_{K, \sigma}^{n+1}\right) N_{K}^{n+1}-B\left(D V_{K, \sigma}^{n+1}\right) N_{L}^{n+1}\right) .
$$

We now prove that

$$
\mathscr{R}_{K, \sigma}^{n+1} \leq \mathscr{S}_{K, \sigma}^{n+1}:=-\min \left(N_{K}^{n+1}, N_{L}^{n+1}\right)\left(D \log \left(N^{n+1}\right)_{K, \sigma}-D V_{K, \sigma}^{n+1}\right)^{2} .
$$


Indeed, applying the property $B(-x)-B(x)=x$, we obtain

$$
\begin{aligned}
& \mathscr{R}_{K, \sigma}^{n+1}-\mathscr{S}_{K, \sigma}^{n+1}=\left(D \log \left(N^{n+1}\right)_{K, \sigma}-D V_{K, \sigma}^{n+1}\right) \times \\
& {\left[\left(B\left(-D V_{K, \sigma}^{n+1}\right)-B\left(-D \log \left(N^{n+1}\right)_{K, \sigma}\right)\right)\left(N_{K}^{n+1}-\min \left(N_{K}^{n+1}, N_{L}^{n+1}\right)\right)\right.} \\
& -\left(B\left(D V_{K, \sigma}^{n+1}\right)-B\left(D \log \left(N^{n+1}\right)_{K, \sigma}\right)\right)\left(N_{L}^{n+1}-\min \left(N_{K}^{n+1}, N_{L}^{n+1}\right)\right) \\
& \left.+B\left(-D \log \left(N^{n+1}\right)_{K, \sigma}\right) N_{K}^{n+1}-B\left(D \log \left(N^{n+1}\right)_{K, \sigma}\right) N_{L}^{n+1}\right]
\end{aligned}
$$

Now, since $B$ is non-increasing on $\mathbb{R}$, the two first terms are non positive, and by using the definition (8) of $B$, the third term is equal to zero. Then we can conclude that

$$
T_{1} \leq \Delta t \sum_{\substack{\sigma \in \mathscr{E}_{i n t} \\ \sigma=K \mid L}} \tau_{\sigma} \mathscr{S}_{K, \sigma}^{n+1}+\Delta t \sum_{K \in \mathscr{T}} \sum_{\sigma \in \mathscr{E}_{\text {ext }, K}^{D}} \tau_{\sigma} \mathscr{S}_{K, \sigma}^{n+1},
$$

and we obtain in the same way a similar estimate for $T_{2}$. To sum up, we have

$$
\mathscr{E}^{n+1}-\mathscr{E}^{n} \leq T_{1}+T_{2} \leq-\Delta t \mathscr{I}^{n+1},
$$

which completes the proof.

\section{Numerical experiments}

We present here a test case for a geometry corresponding to a PN-junction in 1D. The doping profile is piecewise constant, equal to +1 in the N-region $] 0.5,1[$ and -1 in the P-region ] $0,0.5$ [. The Debye length is $\lambda=10^{-2}$.

In Figure 1 we compare the relative energy $\mathscr{E}^{n}$ and its dissipation $\mathscr{I}^{n}$ obtained with the the Scharfetter-Gummel scheme (5) and with the scheme studied by C. Chainais-Hillairet, J. G. Liu and Y. J. Peng in [3], where the diffusion terms are discretized classically and the convection terms are discretized with upwind fluxes. With the Scharfetter-Gummel scheme, we observe that $\mathscr{E}^{n}$ and $\mathscr{I}^{n}$ converge to zero when $n \rightarrow \infty$, which is in keeping with Theorem 2. On the contrary, the upwind scheme, which does not preserve thermal equilibrium, is not very satisfying to reflect the long time behavior of the solution.

Acknowledgements: The author is partially supported by the European Research Council ERC Starting Grant 2009, project 239983-NuSiKiMo. 

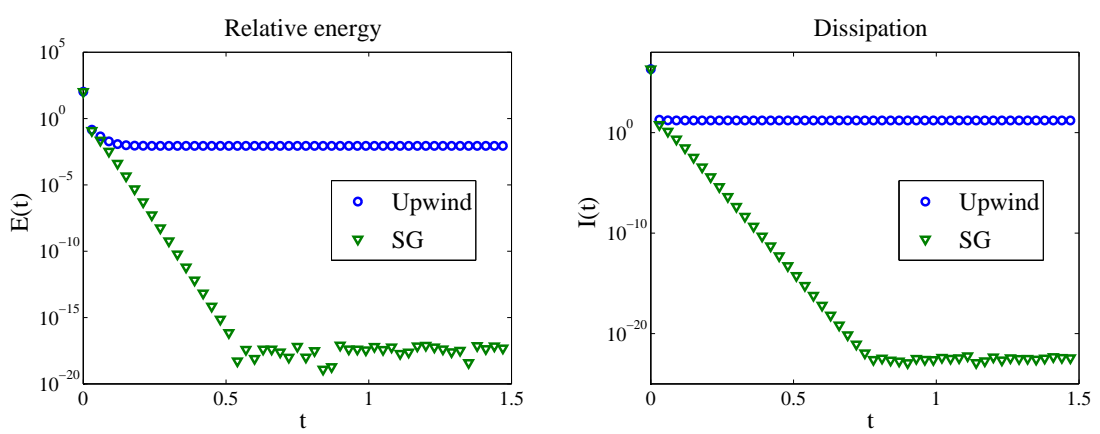

Fig. 1 Evolution of the relative energy $\mathscr{E}^{n}$ and its dissipation $\mathscr{I}^{n}$ in log-scale.

\section{References}

1. F. Brezzi, L.D. Marini, S. Micheletti, P. Pietra, R. Sacco, and S. Wang. Discretization of semiconductor device problems. I. In Handbook of numerical analysis. Vol. XIII, pages 317441. North-Holland, Amsterdam, 2005.

2. C. Chainais-Hillairet and F. Filbet. Asymptotic behavior of a finite volume scheme for the transient drift-diffusion model. IMA J. Numer. Anal., 27(4):689-716, 2007.

3. C. Chainais-Hillairet, J.G. Liu, and Y.J. Peng. Finite volume scheme for multi-dimensional drift-diffusion equations and convergence analysis. M2AN Math. Model. Numer. Anal., 37(2):319-338, 2003.

4. R. Eymard, T. Gallouët, and R. Herbin. Finite volume methods. In Handbook of numerical analysis, volume VII, pages 713-1020. North-Holland, Amsterdam, 2000.

5. H. Gajewki and K. Gärtner. On the discretization of Van Roosbroeck's equations with magnetic field. Z. Angew. Math. Mech., 76(5):247-264, 1996.

6. A. Glitzky. Exponential decay of the free energy for discretized electro-reaction-diffusion systems. Nonlinearity, 21(9):1989-2009, 2008.

7. A.M. Il'in. A difference scheme for a differential equation with a small parameter multiplying the highest derivative. Math. Zametki, 6:237-248, 1969.

8. A. Jüngel. Qualitative behavior of solutions of a degenerate nonlinear drift-diffusion model for semiconductors. Math. Models Methods Appl. Sci., 5(5):497-518, 1995.

9. R. D. Lazarov, Ilya D. Mishev, and P. S. Vassilevski. Finite volume methods for convectiondiffusion problems. SIAM J. Numer. Anal., 33(1):31-55, 1996.

10. D.L. Scharfetter and H.K. Gummel. Large signal analysis of a silicon Read diode. IEEE Trans. Elec. Dev., 16:64-77, 1969.

The paper is in final form and no similar paper has been or is being submitted elsewhere. 